\title{
Universality in the Three-Dimensional Random-Field Ising Model
}

\author{
Nikolaos G. Fytas ${ }^{1,2}$ and Víctor Martín-Mayor ${ }^{1,3}$ \\ ${ }^{1}$ Departamento de Física Teórica I, Universidad Complutense, E-28040 Madrid, Spain \\ ${ }^{2}$ Applied Mathematics Research Centre, Coventry University, Coventry CV1 5FB, United Kingdom \\ ${ }^{3}$ Instituto de Biocomputación and Física de Sistemas Complejos (BIFI), E-50009 Zaragoza, Spain
}

(Received 20 March 2013; published 29 May 2013)

\begin{abstract}
We solve a long-standing puzzle in statistical mechanics of disordered systems. By performing a high-statistics simulation of the $D=3$ random-field Ising model at zero temperature for different shapes of the random-field distribution, we show that the model is ruled by a single universality class. We compute the complete set of critical exponents for this class, including the correction-to-scaling exponent, and we show, to high numerical accuracy, that scaling is described by two independent exponents. Discrepancies with previous works are explained in terms of strong scaling corrections.
\end{abstract}

DOI: 10.1103/PhysRevLett.110.227201

PACS numbers: 75.10.Nr, 02.60.Pn, 75.50.Lk

The random-field Ising model (RFIM) is one of the simplest and most investigated models for collective behavior in the presence of quenched disorder [1]. In spite of its simplicity, many problems in condensed matter physics can be studied through the RFIM: diluted antiferromagnets in a field [2], colloid-polymer mixtures [3,4], colossal magnetoresistance oxides [5,6] (more generally, phase coexistence in the presence of quenched disorder [7-9]), and nonequilibrium phenomena such as the Barkhausen noise in magnetic hysteresis $[10,11]$ or the design of switchable magnetic domains [12], etc.

On the theoretical side, a scaling picture is available [13-16]. The paramagnetic-ferromagnetic phase transition is ruled by a fixed point [in the renormalization-group (RG) sense] at temperature $T=0$ [1]. The spatial dimension $D$ is replaced by $D-\theta$ in hyperscaling relations $(\theta \approx D / 2)$. Nevertheless, many expect only two independent exponents $[1,17,18]$, as in standard phase transitions (see, e.g., [19]).

Unfortunately, establishing the scaling picture is far from trivial. Perturbation theory predicts that, in $D=3$, the ferromagnetic phase disappears upon applying the tiniest random field [20]. Even if the statement holds at all orders in perturbation theory [21], the ferromagnetic phase is stable in $D=3$ [22]. Nonperturbative phenomena are obviously at play $[23,24]$. Indeed, it has been suggested that the scaling picture breaks down because of spontaneous supersymmetry breaking, implying that more than two critical exponents are needed to describe the phase transition [25].

On the experimental side, a particularly well-researched realization of the RFIM is the diluted antiferromagnet in an applied magnetic field [2]. Yet, there are inconsistencies in the determination of critical exponents. In neutron scattering, different parametrizations of the scattering line shape yield mutually incompatible estimates of the thermal critical exponent, namely $\nu=0.87(7)$ [26] and $\nu=1.20(5)$ [27]. Moreover, the anomalous dimension $\eta=0.16(6)$ [26] violates hyperscaling bounds (at least if one believes experimental claims of a divergent specific heat $[28,29])$. Clearly, a reliable parametrization of the line shape would be welcome. This program has been carried out for simpler, better understood problems [30]. Unfortunately, we do not have such a strong command over the RFIM universality class.

The RFIM also has been investigated by means of numerical simulations. However, typical Monte Carlo schemes get trapped into local minima with escape time exponential in $\xi^{\theta}$ ( $\xi$ is the correlation length). Although sophisticated improvements have appeared [31-33], these simulations produced low-accuracy data because they were limited to linear sizes $L \leq 32$. Larger sizes can be achieved at $T=0$, through the well-known mapping of the ground state to the maximum-flow optimization problem [34-44]. Yet, $T=0$ simulations lack many tools, standard at $T>0$. In fact, the numerical data at $T=0$ and their finite-size scaling analysis mostly resulted in strong universality violations [33,36-39].

Here we show that the $D=3$ RFIM is ruled by a single universality class, by considering explicitly four different models that belong to it. To this end, we perform highstatistics $T=0$ simulations of the model, and we introduce a fluctuation-dissipation formalism in order to compute connected and disconnected correlation functions. Another asset of our implementation is the use of phenomenological renormalization $[45,46]$, which allows us to extract effective size-dependent critical exponents, whose size evolution can be closely followed. Although the four models differ in their prediction for finite sizes, we show that, after a proper consideration of the scaling corrections, we can extrapolate to infinite-limit size, finding consistent results for all of them.

Our $S_{x}= \pm 1$ spins are on a cubic lattice with size $L$ and periodic boundary conditions. The Hamiltonian is

$$
\mathcal{H}=-J \sum_{\langle x, y\rangle} S_{x} S_{y}-\sum_{x} h_{x} S_{x} .
$$


$J=1$ is the nearest-neighbors' ferromagnetic interaction. Independent quenched random fields $h_{x}$ are extracted from one of the following double Gaussian (dG) or Poissonian (P) distributions (with parameters $h_{R}, \sigma$ ),

$$
\begin{aligned}
\mathrm{dG}^{(\sigma)}\left(h_{x} ; h_{R}\right)= & \frac{1}{\sqrt{8 \pi \sigma^{2}}}\left[e^{-\left(\left(h_{x}-h_{R}\right)^{2} / 2 \sigma^{2}\right)}\right. \\
& \left.+e^{-\left(\left(h_{x}+h_{R}\right)^{2} / 2 \sigma^{2}\right)}\right] \\
\mathrm{P}\left(h_{x} ; \sigma\right) & =\frac{1}{2|\sigma|} e^{-\left|h_{x}\right| / \sigma}
\end{aligned}
$$

The limiting cases $\sigma=0$ and $h_{R}=0$ of Eq. (2) correspond to the well-known bimodal (b) and Gaussian (G) distributions, respectively. In the $\mathrm{P}$ and $\mathrm{G}$ cases the strength of the random fields is parametrized by $\sigma$, while in the $\mathrm{dG}$ case we shall take $\sigma=1$ and 2 and vary $h_{R}$. The phase diagram for the double Gaussian distribution is sketched in Fig. 1. Note the bimodal shape of Eq. (2) for $\sigma=1$, with peaks near $\pm h_{R}$.

An instance of the random fields $\left\{h_{x}\right\}$ is named a sample. The only relevant spin configurations at $T=0$ are ground states, which are nondegenerate for continuous randomfield distributions [47]. Thermal mean values are denoted as $\langle\cdots\rangle$. The subsequent average over samples is indicated by an overline (e.g., for the magnetization density $m=\sum_{x} s_{x} / L^{D}$, we consider both $\langle m\rangle$ and $\overline{\langle m\rangle}$ ).

We considered four disorder distributions: $\mathrm{P}, \mathrm{G}$, and $\mathrm{dG}$ with $\sigma=1,2$. We obtained the ground states using the push-relabel algorithm [48]. We implemented in C the algorithm in [41,42], with periodic global updates. Our lattice sizes were $L=12,16,24,32,48,64,96,128$, and $192\left[16 \leq L \leq 128\right.$ for $\mathrm{dG}^{(\sigma=1)}$ and $12 \leq L \leq 128$ for $\left.\mathrm{dG}^{(\sigma=2)}\right]$. For each $L$, we averaged over $10^{7}$ samples

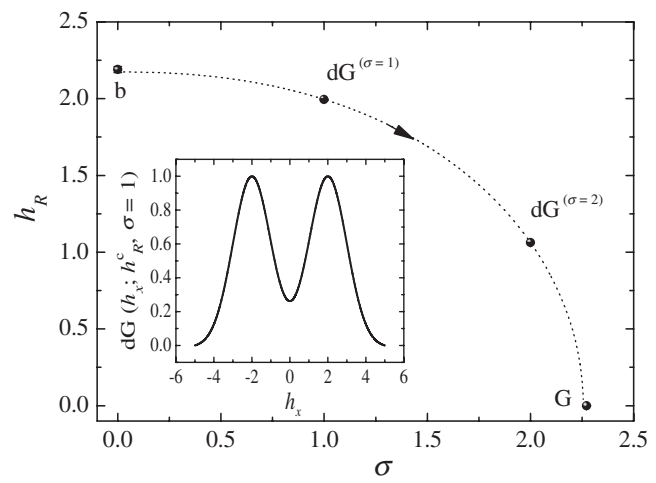

FIG. 1. Phase diagram of the double Gaussian RFIM, Eq. (2), at $T=0$. The dotted critical line (a simple guide to the eye) separates the paramagnetic phase (large $\sigma, h_{R}$ ) from the ordered phase (low $\sigma, h_{R}$ ). Transition points are computed here (data from [54]), but for the limit $\sigma=0$ which corresponds to binary random fields [39]. The arrow along the critical line indicates the RG flow. Inset: bimodal shape of the critical double Gaussian distribution (2) with $\sigma=1$. $\left[5 \times 10^{7}\right.$ samples for $\left.\mathrm{dG}^{(\sigma=1)}\right]$. Previous studies were limited to $\sim 10^{4}$ samples [40,41].

We have generalized the fluctuation-dissipation formalism of [49] to compute connected $G_{x y}=\overline{\partial\left[\left\langle S_{x}\right\rangle\right] / \partial h_{y}}$ and disconnected $G_{x y}^{(\text {dis })}=\overline{\left\langle S_{x} S_{y}\right\rangle}$ correlation functions. We compute from them the second-moment connected $(\xi)$ and disconnected $\left(\xi^{\text {(dis) }}\right)$ correlation lengths $[19,50]$.

We have also extended reweighting methods from percolation studies [51,52]. From a single simulation, we extrapolate the mean value of observables to nearby parameters of the disorder distribution [we varied $\sigma$ for the G and $\mathrm{P}$ distributions (see Fig. 2) and $h_{R}$ for the dG case]. Computing derivatives with respect to $\sigma$ or $h_{R}$ is straightforward. Consider, for instance, the $\mathrm{P}$ case (see [53] for other distributions). Let $\mathcal{D}=\sum_{x}\left(\left|h_{x}\right|-\sigma\right) / \sigma^{2}$. The connected correlation function is $\left.G_{x y}=\overline{h_{y}\left\langle S_{x}\right\rangle /\left[\left|h_{y}\right| \sigma\right)}\right]$, while the $\sigma$ derivative and the reweighting extrapolation to $\sigma+\delta \sigma$ of a generic observable $O$ are

$$
\begin{aligned}
D_{\sigma}{\overline{\langle O\rangle_{\sigma}}} & ={\overline{\langle O \mathcal{D}\rangle_{\sigma}}}, \quad{\overline{\langle O\rangle_{\sigma+\delta \sigma}}}_{\overline{\langle O \mathcal{R}\rangle_{\sigma}}} \text { with } \\
\mathcal{R} & =\exp \left[\mathcal{D} \frac{\sigma \delta \sigma}{\sigma+\delta \sigma}+L^{D}\left(\log \frac{\sigma}{\sigma+\delta \sigma}+\frac{\sigma+\delta \sigma}{\sigma}\right)\right] .
\end{aligned}
$$

To extract the value of critical points, critical exponents, and dimensionless quantities, we employ the quotients method $[19,45,46]$. We compare observables computed in a pair of lattices $(L, 2 L)$. We start imposing scale invariance by seeking the $L$-dependent critical point: the value of $\sigma\left(h_{R}\right.$ for the $\left.\mathrm{dG}\right)$, such that $\xi_{2 L} / \xi_{L}=2$ [i.e., the crossing point for $\xi_{L} / L$, (see Fig 2)]. Now, for dimensionful quantities $O$, scaling in the thermodynamic limit as $\xi^{x_{O} / \nu}$, we

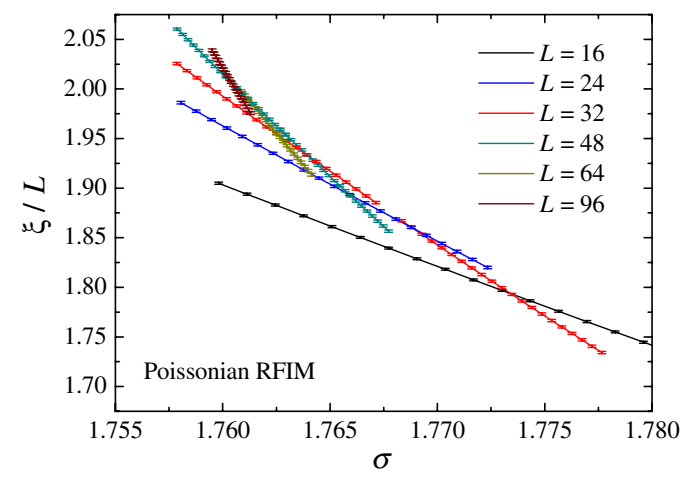

FIG. 2 (color online). For several system sizes, we show $\xi / L$ vs the strength of the Poissonian random field $\sigma$ [see Eq. (3)] ( $\xi$ : correlation length from the connected correlator, $L$ : system size). Lines join data obtained from reweighting extrapolation, Eq. (4) (discontinuous lines of the same color come from independent simulations). In the large- $L$ limit, $\xi / L$ is $L$ independent at the critical point $\sigma^{c}$. In the quotients method, we consider the $\xi / L$ curves for a pair of lattices $(L, 2 L)$ and seek the $\sigma$ where they cross. This crossing is employed for computing effective, $L$-dependent critical exponents with Eq. (5). 
TABLE I. For our four field distributions, size-dependent critical exponents of the $D=3$ RFIM as computed from the quotients method.

\begin{tabular}{lclcr}
\hline \hline Distribution & $\left(L_{1}, L_{2}\right)$ & \multicolumn{1}{c}{$\nu$} & \multicolumn{1}{c}{$\eta$} & $2 \eta-\bar{\eta}$ \\
\hline $\mathrm{G}$ & $(16,32)$ & $1.48(3)$ & $0.5168(6)$ & $0.0038(11)$ \\
& $(24,48)$ & $1.45(3)$ & $0.5155(5)$ & $0.0022(11)$ \\
& $(32,64)$ & $1.36(4)$ & $0.5150(5)$ & $0.0019(10)$ \\
& $(48,96)$ & $1.43(6)$ & $0.5154(5)$ & $0.0033(9)$ \\
& $(64,128)$ & $1.38(9)$ & $0.5142(5)$ & $0.0014(10)$ \\
$\mathrm{dG}^{(\sigma=1)}$ & $(96,192)$ & $1.38(11)$ & $0.5144(5)$ & $0.0021(11)$ \\
& $(16,32)$ & $3.04(14)$ & $0.5035(7)$ & $0.0016(15)$ \\
& $(24,48)$ & $2.26(9)$ & $0.5083(7)$ & $0.0034(14)$ \\
& $(32,64)$ & $1.87(8)$ & $0.5093(7)$ & $0.0010(13)$ \\
$\mathrm{dG}^{(\sigma=2)}$ & $(48,96)$ & $1.56(9)$ & $0.5121(7)$ & $0.0026(14)$ \\
& $(64,128)$ & $1.67(12)$ & $0.5125(8)$ & $0.0015(17)$ \\
& $(16,32)$ & $1.48(5)$ & $0.5154(6)$ & $0.0020(12)$ \\
& $(24,48)$ & $1.50(6)$ & $0.5151(7)$ & $0.0020(13)$ \\
& $(32,64)$ & $1.41(8)$ & $0.5142(7)$ & $0.0004(13)$ \\
$\mathrm{P}$ & $(48,96)$ & $1.36(10)$ & $0.5148(7)$ & $0.0024(14)$ \\
& $(64,128)$ & $1.31(11)$ & $0.5154(6)$ & $0.0041(13)$ \\
& $(16,32)$ & $1.20(2)$ & $0.5183(9)$ & $-0.0006(19)$ \\
& $(24,48)$ & $1.26(3)$ & $0.5168(8)$ & $0.0011(17)$ \\
& $(32,64)$ & $1.30(4)$ & $0.5153(8)$ & $0.0005(17)$ \\
& $(48,96)$ & $1.37(7)$ & $0.5143(9)$ & $0.0004(18)$ \\
& $(64,128)$ & $1.33(7)$ & $0.5148(8)$ & $0.0024(16)$ \\
& $(96,192)$ & $1.43(13)$ & $0.5146(8)$ & $0.0026(17)$ \\
\hline & & & & \\
& & & & \\
& & & &
\end{tabular}

consider the quotient $Q_{O}=O_{2 L} / O_{L}$ at the crossing. For dimensionless magnitudes $g$, we focus on $g_{2 L}$. In either case, one has

$$
Q_{O}^{\text {cross }}=2^{x_{O} / \nu}+\mathcal{O}\left(L^{-\omega}\right), \quad g_{(2 L)}^{\text {cross }}=g^{*}+\mathcal{O}\left(L^{-\omega}\right),
$$

where $x_{O} / \nu, g^{*}$ and the scaling-corrections exponent $\omega$ are universal. Examples of dimensionless quantities are $\xi / L$, $\xi^{\text {(dis) }} / L$, and $U_{4}=\overline{\left\langle m^{4}\right\rangle} / \overline{\left\langle m^{2}\right\rangle^{2}}$. Instances of dimensionful quantities are the derivatives of $\xi$, $\xi^{\text {(dis) }}\left(x_{\xi}=1+\nu\right)$, the connected and disconnected susceptibilities $\chi$ and $\chi^{(\mathrm{dis})}$ $\left[x_{\chi}=\nu(2-\eta), x_{\chi^{\text {(dis) }}}=\nu(4-\bar{\eta})\right]$, and the ratio $U_{22}=$ $\chi^{(\mathrm{dis})} / \chi^{2}\left[x_{U_{22}}=\nu(2 \eta-\bar{\eta})\right]$.

The application of Eq. (5) to our four random-field distributions is summarized in Table I and Figs. 3 and 4 (the numerical values are tabulated in [54]). We start inspecting $\xi / L$ in Fig. 3. At fixed $L$, the dependence on the distribution is substantial. However, the strong size evolution suggests a common $L \rightarrow \infty$ limit. The behavior of the critical exponents, $\xi^{\text {(dis) }} / L$ and $U_{4}$, is similar.

In order to extrapolate to $L \rightarrow \infty$, one fits the data of Table I to polynomials in $L^{-\omega}$. Although the procedure is standard [52], it has not been attempted before for the RFIM. Our extrapolations are documented in Fig. 4 and Table II. The following comments are in order: (a) For dimensionless quantities we needed a third-order polynomial in $L^{-\omega}$ (only a subclass of the subleading corrections-to-scaling [19]).

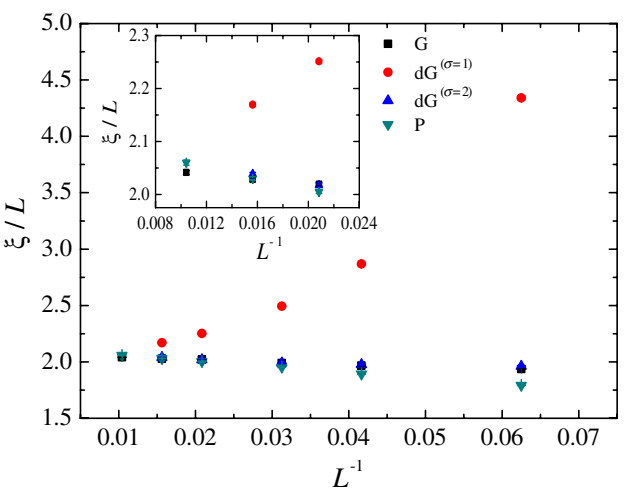

FIG. 3 (color online). Inspection of the size dependence of universal quantities. We show the universal ratio $\xi / L$ vs $1 / L$, as computed at the corresponding crossing points (see Fig. 2) for the four disorder distributions considered in this work. The inset is an enlargement for $L \geq 48$.

Leading-order corrections sufficed for critical exponents. (b) We are not aware of any other computation of $\omega, \xi / L$, $\xi^{\text {(dis) }} / L$, and $U_{4}$. All of them are universal. (c) The full critical line belongs to a single universality class (justifying

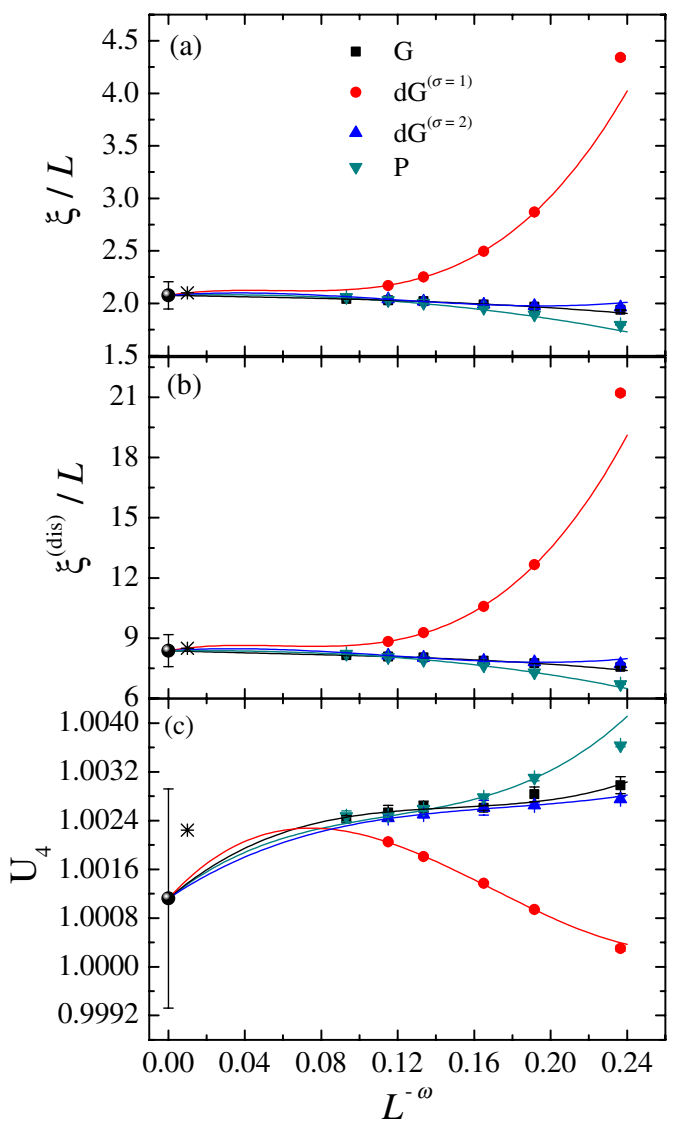

FIG. 4 (color online). Computation of the corrections-toscaling exponent $\omega$ [see Eq. (5)] by means of a joint fit for $\xi / L(a), \xi^{(\mathrm{dis})} / L(b)$, and $U_{4}=\overline{\left\langle m^{4}\right\rangle} / \overline{\left\langle m^{2}\right\rangle^{2}}(c)$; see Table II and [63]. Black circles depicted at $L^{-\omega}=0$ are our extrapolations to $L=\infty$. Stars denote extrapolations obtained using only the diagonal terms of the covariance matrix. 
TABLE II. Extrapolations to $L=\infty$ for the critical $\xi / L$, $\xi^{\text {(dis) }} / L, U_{4}$ and the exponents of the $D=3$ RFIM, (finite- $L$ data are listed in [54]). We perform joint fits for sizes $L \geq L_{\min }$ to polynomials in $L^{-\omega}$, imposing common extrapolations for all four random-field distributions. The $\chi^{2}$ figure of merit was computed with the full covariance matrix (DOF: number of degrees of freedom in the fit). For the exponent $\nu$, we considered derivatives of both $\xi$ and $\xi^{(\mathrm{dis})}$. The error induced by the uncertainty in $\omega$ is given as a second error estimate. The extrapolation of the critical points slightly differs [55].

\begin{tabular}{lccc}
\hline \hline Extrapolation & $\chi^{2} / \mathrm{DOF}$ & $L_{\min }$ & Order in $L^{-\omega}$ \\
\hline$\left.(\xi / L)\right|_{L=\infty}=2.08(13)$ & $18.8 / 14$ & 24 & third \\
$\left.\left(\xi^{(\mathrm{dis})} / L\right)\right|_{L=\infty}=8.4(8)$ & & & \\
$\left.U_{4}\right|_{L=\infty}=1.0011(18)$ & & & \\
$\omega=0.52(11)$ & & & \\
$\left.\nu\right|_{L=\infty}=1.38(10)(0.03)$ & $12.5 / 10$ & 32 & first \\
$\left.\eta\right|_{L=\infty}=0.5153(9)(2)$ & $10.0 / 9$ & 32 & first \\
$\left.(2 \eta-\bar{\eta})\right|_{L=\infty}=0($ fixed $)$ & $18.3 / 18$ & 16 & first \\
$\left.(2 \eta-\bar{\eta})\right|_{L=\infty}=0.0026(9)(1)$ & $10.5 / 17$ & 16 & first \\
$\sigma^{c}[\mathrm{G}]=2.27205(18)(4)$ & $3.1 / 3$ & 16 & second \\
$h_{R}^{c}[\mathrm{dG}(\sigma=1)]=1.9955(6)(24)$ & $2.5 / 1$ & 24 & second \\
$h_{R}^{c}[\mathrm{dG}(\sigma=2)]=1.0631(7)(10)$ & $0.7 / 2$ & 16 & second \\
$\sigma^{c}[\mathrm{P}]=1.7583(2)(2)$ & $3.0 / 3$ & 16 & second \\
\hline \hline
\end{tabular}

a posteriori the RG flow in Fig. 1). The fixed point at $\sigma=0$ (bimodal RFIM) [36-39] has no basin of attraction. (d) The two-exponent scaling scenario [1] $(2 \eta=\bar{\eta})$ is supported by our data. Violations are at most $\sim 0.002$, much smaller than predicted in [25]. (e) Our $\nu$ estimation is similar to modern computations [40,42-44]. For the anomalous dimension, we note $\eta=0.50$ (3) [40]. With some caution, we quote as well [56]. Our errors for $\nu$ are larger than for $\eta$ or $\bar{\eta}$ because we compute derivatives as connected correlations, Eq. (4). (f) The two-exponent scenario implies for the hyperscaling violation exponent $\theta=2-\eta=1.4847$ (11) (recall $\theta=1.469(20)$ [31] or 1.49(3) [41]). (g) For the Gaussian RFIM, our critical point compares favorably to the value 2.270(4) of Ref. [41].

Where do we stand, at this point? Clearly, our confirmation of Universality provides motivation to undertake an ambitious field-theoretic study of the RFIM. The task is difficult, though. The direct approach of Refs. [20,21] fails because the supersymmetry is spontaneously broken as soon as $\theta \neq 2$. The phenomenological two-exponent scaling picture $[17,18]$ seems highly successful, but it has not yet been derived from field theory. However, it does suggest a systematic way of computing critical exponents: study the ferromagnetic Ising model in the absence of disorder, but with a noninteger spatial dimension $d^{\prime}=$ $D-\theta$ [18] $\left(d^{\prime} \approx 1.5153\right.$ (11) according to our results for $D=3)$. The computation has been attempted within the $\epsilon$ expansion, where $\epsilon=4-d^{\prime}$. The results up to order $\epsilon^{5}$ are encouraging, but not very accurate [57]. On the other hand, a first-principles approach, the functional renormalization group (fRG) [58], does explain the spontaneous breaking of the supersymmetry [25]. Yet, the fRG prediction for the critical exponents is rather crude. Furthermore, the smallness of $2 \eta-\bar{\eta}$ (see Table II) remains unexplained. Fortunately, fRG computations can be systematically improved through the parametrization of the effective action. In this way, high accuracy has been reached for nondisordered systems [59]. Refinements are probably feasible as well for the RFIM [60].

In conclusion, we have shown that the universality class of the RFIM is independent of the form of the implemented random-field distribution, in disagreement with the current opinion in the literature $[32,33,36-39]$ and with the early predictions of mean-field theory [61]. To reach this conclusion, we had to identify and control the role of scaling corrections, the Achilles heel in the study of the RFIM (this problem was emphasized in the pioneering work of [34], but it was overlooked in subsequent investigations). On technical parts, we have developed a fluctuation-dissipation formalism that allowed us to compute correlation functions and to apply phenomenological renormalization. We have also adapted the approach of [62] to study the scaling of the energy, checking that our data are compatible with modified hyperscaling [53,54] (a rather slippery problem [40]). Hence, several contradictions of previous works have been resolved in a consistent picture, paving the way to more sophisticated, experimentally relevant computations.

We were partly supported by MICINN, Spain, through research contracts No. FIS2009-12648-C03 and No. FIS2012-35719-C02-01. Significant allocations of computing time were obtained in the clusters Terminus and Memento (BIFI). We are grateful to D. Yllanes and, especially, to L. A. Fernández for substantial help during several parts of this work. We also thank A. Pelissetto and G. Tarjus for useful correspondence.

[1] See T. Nattermann in [64].

[2] See D. P. Belanger in [64].

[3] R. L. C. Vink, K. Binder, and H. Löwen, Phys. Rev. Lett. 97, 230603 (2006).

[4] M. A. Annunziata and A. Pelissetto, Phys. Rev. E 86, 041804 (2012).

[5] E. Dagotto, Science 309, 257 (2005).

[6] J. Burgy, M. Mayr, V. Martín-Mayor, A. Moreo, and E. Dagotto, Phys. Rev. Lett. 87, 277202 (2001).

[7] J. Cardy and J. L. Jacobsen, Phys. Rev. Lett. 79, 4063 (1997).

[8] L. A. Fernández, A. Gordillo-Guerrero, V. Martín-Mayor, and J. J. Ruiz-Lorenzo, Phys. Rev. Lett. 100, 057201 (2008).

[9] L. A. Fernández, A. Gordillo-Guerrero, V. Martín-Mayor, and J. J. Ruiz-Lorenzo, Phys. Rev. B 86, 184428 (2012).

[10] J. P. Sethna, K. Dahmen, S. Kartha, J. A. Krumhansl, B. W. Roberts, and J. D. Shore, Phys. Rev. Lett. 70, 3347 (1993).

[11] O. Perković, K. A. Dahmen, and J. P. Sethna, Phys. Rev. B 59, 6106 (1999).

[12] D. M. Silevitch, G. Aeppli, and T.F. Rosenbaum, Proc. Natl. Acad. Sci. U.S.A. 107, 2797 (2010).

[13] Y. Imry and S.-K. Ma, Phys. Rev. Lett. 35, 1399 (1975). 
[14] J. Villain, Phys. Rev. Lett. 52, 1543 (1984).

[15] A. J. Bray and M. A. Moore, J. Phys. C 18, L927 (1985).

[16] D. S. Fisher, Phys. Rev. Lett. 56, 416 (1986).

[17] A. Aharony, Y. Imry, and S.-K. Ma, Phys. Rev. Lett. 37, 1364 (1976).

[18] M. Gofman, J. Adler, A. Aharony, A. B. Harris, and M. Schwartz, Phys. Rev. Lett. 71, 1569 (1993).

[19] See, e.g., D. Amit and V. Martín-Mayor, Field Theory, the Renormalization Group and Critical Phenomena (World Scientific, Singapore, 2005), 3rd ed.

[20] A. P. Young, J. Phys. A 10, L257 (1977).

[21] G. Parisi and N. Sourlas, Phys. Rev. Lett. 43, 744 (1979).

[22] J. Bricmont and A. Kupiainen, Phys. Rev. Lett. 59, 1829 (1987).

[23] G. Parisi, Field Theory, Disorder and Simulations (World Scientific, Singapore, 1994).

[24] G. Parisi and N. Sourlas, Phys. Rev. Lett. 89, 257204 (2002).

[25] M. Tissier and G. Tarjus, Phys. Rev. Lett. 107, 041601 (2011); Phys. Rev. B 85, 104203 (2012).

[26] Z. Slanič, D. P. Belanger, and J. A. Fernandez-Baca, Phys. Rev. Lett. 82, 426 (1999).

[27] F. Ye, M. Matsuda, S. Katano, H. Yoshizawa, D. P. Belanger, E. T. Seppälä, J. A. Fernandez-Baca, and M. J. Alava, J. Magn. Magn. Mater. 272-276, 1298 (2004).

[28] D. P. Belanger, A. R. King, V. Jaccarino, and J. L. Cardy, Phys. Rev. B 28, 2522 (1983).

[29] D. P. Belanger and Z. Slanič, J. Magn. Magn. Mater. 186, 65 (1998).

[30] V. Martín-Mayor, A. Pelissetto, and E. Vicari, Phys. Rev. E 66, 026112 (2002).

[31] L. A. Fernández, V. Martín-Mayor, and D. Yllanes, Phys. Rev. B 84, 100408 (2011).

[32] N. G. Fytas, A. Malakis, and K. Eftaxias, J. Stat. Mech.: Theory Exp. (2008) P03015.

[33] B. Ahrens, J. Xiao, A. K. Hartmann, and H. G. Katzgraber, arXiv:1302.2480.

[34] A. T. Ogielski, Phys. Rev. Lett. 57, 1251 (1986).

[35] J.-C. Anglès d Auriac, Ph.D. thesis, Centre de Recherches sur les Très Basses Températures, Grenoble, France, 1986.

[36] N. Sourlas, Comput. Phys. Commun. 121-122, 183 (1999).

[37] J.-C. Anglès Auriac and N. Sourlas, Europhys. Lett. 39, 473 (1997)

[38] M. R. Swift, A. J. Bray, A. Maritan, M. Cieplak, and J. R. Banavar, Europhys. Lett. 38, 273 (1997).

[39] A. K. Hartmann and U. Nowak, Eur. Phys. J. B 7, 105 (1999).

[40] A. K. Hartmann and A. P. Young, Phys. Rev. B 64, 214419 (2001).

[41] A. A. Middleton and D. S. Fisher, Phys. Rev. B 65, 134411 (2002).
[42] A. A. Middleton, Phys. Rev. Lett. 88, 017202 (2001).

[43] I. Dukovski and J. Machta, Phys. Rev. B 67, 014413 (2003).

[44] Y. Wu and J. Machta, Phys. Rev. Lett. 95, 137208 (2005); Phys. Rev. B 74, 064418 (2006).

[45] H. G. Ballesteros, L. A. Fernández, V. Martín-Mayor, and A. Muñoz-Sudupe, Phys. Lett. B 378, 207 (1996).

[46] M. P. Nightingale, Physica (Amsterdam) 83A, 561 (1976).

[47] Severe ground-state degeneracy arises for discrete distributions, which needs to be taken into account; see S. Bastea and P. M. Duxbury, Phys. Rev. E 58, 4261 (1998).

[48] A. V. Goldberg and R. E. Tarjan, J. Assoc. Comput. Mach. 35, 921 (1988).

[49] For instance, in the Gaussian RFIM $G_{x y}=\overline{h_{y}\left\langle S_{x}\right\rangle} / \sigma^{2}$, see M. Schwartz and A. Soffer, Phys. Rev. Lett. 55, 2499 (1985).

[50] F. Cooper, B. Freedman, and D. Preston, Nucl. Phys. B210, 210 (1982).

[51] G. Harris, Nucl. Phys. B418, 278 (1994).

[52] H. G. Ballesteros, L. A. Fernández, V. Martín-Mayor, A. MuñozSudupe, G. Parisi, and J. J. Ruiz-Lorenzo, Phys. Rev. B 58, 2740 (1998); Nucl. Phys. B512, 681 (1998); J. Phys. A 30, 8379 (1997); Phys. Lett. B 400, 346 (1997).

[53] N. G. Fytas and V. Martín-Mayor (to be published).

[54] See Supplemental Material at http://link.aps.org/ supplemental/10.1103/PhysRevLett.110.227201 for raw results of the quotients method and for the specific-heat exponent.

[55] Scaling corrections for the critical point are of order $L^{-(\omega+(1 / \nu))}, L^{-(2 \omega+(1 / \nu))}$, etc. [19,53].

[56] The two-exponent scaling relation $\eta=(4-D+$ $2 \beta / \nu) / 2$ yields $\eta=0.5111(8)$; see [31]. However, this error is underestimated, as it does not consider scaling corrections nor the uncertainty in the critical value of $\hat{m}$.

[57] T. Jolicoeur and J.-C. Le Guillou, Phys. Rev. B 56, 10766 (1997).

[58] C. Wetterich, Phys. Lett. B 301, 90 (1993).

[59] F. Benitez, J.-P. Blaizot, H. Chaté, B. Delamotte, R. Méndez-Galain, and N. Wschebor, Phys. Rev. E 85, 026707 (2012).

[60] G. Tarjus (private communication).

[61] A. Aharony, Phys. Rev. B 18, 3318 (1978); 18, 3328 (1978); D. Andelman, ibid. 27, 3079 (1983).

[62] C. Holm and W. Janke, Phys. Rev. Lett. 78, 2265 (1997).

[63] Joint fits share the value of some fitting parameters such as the $L \rightarrow \infty$ extrapolation (which is the same for all random-field distributions) or the corrections-to-scaling exponent $\omega$ (which is common to all magnitudes).

[64] Spin Glasses and Random Fields, edited by A. P. Young (World Scientific, Singapore, 1998). 